\title{
ENSEÑAR EN LA SOCIEDAD DE LA INFORMACIÓN Y EL CONOCIMIENTO. CREENCIAS ACERCA DE LA FORMACIÓN INICIAL DEL PROFESORADO DE LENGUAS EXTRANJERAS -INGLÉS Y FRANCÉS- EN EDUCACIÓN INFANTIL
}

\author{
Juana Herrera Cubas \\ María Nieves Pozas Ortega \\ Universidad de La Laguna
}

\begin{abstract}
RESUMEN
Con la firma del Acuerdo de Lisboa en el año 2000, el Consejo de Europa sentó las bases para el establecimiento de una política lingüística común a todos los estados miembros de la Unión Europea. En las casi dos décadas transcurridas desde entonces, una de las medidas más exitosas y que, sin duda, constituye un logro en materia social y de derechos humanos es el adelanto de la edad en que los escolares deberían comenzar el aprendizaje de lenguas extranjeras, que, en la Comunidad Autónoma de Canarias, se extiende al segundo ciclo de Educación Infantil (3-6 años). El estudio que se presenta a continuación se llevó a cabo con dos grupos de estudiantes de 3 . $^{\text {er }}$ curso del Grado de Maestro de Educación Infantil, con la finalidad de investigar sus creencias acerca de la importancia de las lenguas extranjeras en esta etapa y, más concretamente, sus creencias acerca de la necesidad de formar profesorado competente para enseñar inglés y francés a edades tempranas.
\end{abstract}

Palabras clave: formación del profesorado, lenguas extranjeras, educación temprana, competencias profesionales.

\section{TEACHING IN THE INFORMATION AND KNOWLEDGE BASED SOCIETY. BELIEFS ABOUT PRE-SERVICE FOREIGN LANGUAGE TEACHER TRAINING -ENGLISH AND FRENCH-IN PRE-SCHOOL EDUCATION}

\section{Abstract}

The Lisbon Agenda, published in the year 2000, set the conditions for the establishment of the future linguistic policies across the European state members. After two decades of implementation, one of the most successful measures agreed upon, both in terms of social advancement and human rights, concerns early foreign language learning at pre-primary level, which comprises ages three to six in the Canary Islands. This study was carried out with two groups of undergraduate students, in their third year of the Pre-school Education Degree, to investigate their beliefs about the importance of early foreign language learning and, more specifically, about the need to train competent pre-school teachers to teach both English and French in Pre-school Education.

KEYwORDs: teacher education, early foreign language teaching and learning, teacher competences. 


\section{INTRODUCCIÓN}

La firma del Acuerdo de Lisboa en el año 2000 supuso un paso crucial hacia el establecimiento de una política lingüística común a todos los estados miembros de la Unión Europea. Una de las medidas más exitosas que posteriormente se tomarían fue, sin duda, el adelanto de la edad en que los escolares deberían iniciar el aprendizaje de lenguas extranjeras, por los beneficios que esto podría reportar, a largo plazo, en su desarrollo lingüístico y global. Es necesario aclarar, sin embargo, que la publicación de la Resolución del Consejo de la Unión Europea de $1997^{1}$, relativa a la enseñanza precoz de lenguas, ya ponía de manifiesto la conveniencia de enseñar una o varias lenguas distintas de la materna, y la de sensibilizar a los escolares hacia la diversidad lingüística y cultural propia de Europa.

En este marco, la Consejería de Educación, Cultura y Deportes de la Comunidad Autónoma de Canarias puso en marcha la realización de proyectos experimentales de anticipación de la primera lengua extranjera-inglés- en el primer ciclo de la Educación Primaria. Esta misma experiencia se llevaría a cabo, posteriormente, en la Educación Infantil -iniciativa pionera en el Estado español- mediante la Orden de 26 de mayo de 2000, por la que se regulaba, con carácter experimental igualmente, la impartición del inglés en el segundo ciclo de la etapa -3 a 6 años-. La citada orden establecía que el inglés debía ser impartido por el especialista del área, que lo haría también en el primer ciclo de Primaria, en coordinación con el profesorado tutor del segundo ciclo de Educación Infantil.

Finalmente, con la publicación de la Orden de 20 de mayo de 2002, modificada por la Orden de 30 de junio de 2003, y la Resolución de 22 de julio de 2002, la Consejería de Educación dictó instrucciones para la implantación del inglés como primera lengua extranjera obligatoria en el segundo ciclo de Infantil. Seguía reconociéndose la prioridad de impartición al profesorado especialista, excepto en aquellos casos en que el centro contara con un tutor de Educación Infantil que hubiera participado en los proyectos experimentales, y, en segundo lugar, a los tutores de Infantil con la especialidad de inglés. Este orden de prioridad se mantiene a día de hoy. Ese mismo año, también, se anticipó la generalización de una segunda lengua extranjera -francés- en el tercer ciclo de Primaria, única etapa en que se imparte hasta la actualidad, si bien es una formación que se oferta a los alumnos del actual Grado de Educación Infantil.

Desde la publicación de esas órdenes, la Consejería de Educación ha puesto en marcha diversas acciones y proyectos, en línea con las disposiciones y normativa europeas, para fomentar el interés por las lenguas extranjeras a edades tempranas,

\footnotetext{
${ }^{1}$ Comunidad Europea (1997), Resolución del Consejo de la Unión Europea de 16 de diciembre de 1997 relativa a la enseñanza precoz de las lenguas de la Unión Europea, Diario de las Comunidades Europeas, https://eur-/ex.europa.eu/legalcontent/ES/TXT/PDF/?uri=CELEX:31998Y0103(01)\&from=ES.
} 
ya que considera prioritario, en la sociedad de la información y el conocimiento, que la población escolar alcance una competencia comunicativa adecuada al terminar la enseñanza obligatoria. En este sentido, es necesario destacar, por su importancia, el Plan de Impulso de las Lenguas Extranjeras (PILE) de Canarias, dirigido a todas las etapas educativas no universitarias y con un periodo de vigencia desde el curso 2016-17 al 2037-38. En su tercer año de pilotaje, de los seis previstos, el PILE comprende un conjunto de actuaciones con las que se pretende el desarrollo competencial del alumnado en lenguas extranjeras, la continuidad de los proyectos educativos de éxito, la generalización de los programas bilingües existentes y la implantación de acciones encaminadas a garantizar la formación a lo largo de la vida. En definitiva, se plantea como meta la incorporación de todos los centros sostenidos con fondos públicos al PILE y, más importante aún, la impartición de, al menos, $40 \%$ del currículo de todos los niveles educativos en inglés.

Los centros de Educación Infantil de Canarias, entonces, iniciaron la enseñanza del inglés, con carácter obligatorio, tiempo antes de que se comenzara a formar al profesorado de la etapa, formación que proporciona el actual Grado de Maestro, en inglés y francés, desde su implantación en el curso 2010-2011. El Grado, sin embargo, se encuentra en proceso de revisión y, como resultado, se ha decidido eliminar todas las menciones asociadas al mismo en favor de un perfil generalista, una decisión que, obviamente, dará mayor opción de empleabilidad a los egresados de Primaria, en virtud de las disposiciones PILE, ya que estos seguirán contando con la mención de lengua extranjera.

El estudio que se presenta a continuación surgió, por tanto, del interés por conocer la opinión de dos grupos de alumnos que cursaban la materia de Didáctica de la Lengua extranjera, en tercer año del Grado de Infantil, acerca de la importancia de enseñar lenguas extranjeras a edades tempranas. Concretamente, se investigan sus creencias acerca de la necesidad de formar profesorado competente para enseñar inglés y francés en Educación Infantil.

\section{LA SOCIEDAD DE LA INFORMACIÓN \\ Y EL CONOCIMIENTO: DE LISBOA 2000 A EDUCACIÓN Y FORMACIÓN 2020}

Con la firma del Acuerdo de Lisboa, el Consejo de Europa definió el que sería su objetivo estratégico para la década: convertir a la Unión Europea en la «economía basada en el conocimiento más competitiva y dinámica del mundo, capaz de crecer económicamente de manera sostenible, con más y mejores empleos y con mayor cohesión social» (European Council, 2000, p. 12). Para lograr dicho objetivo, en el ámbito de educación y formación, se acordó poner en marcha una estrategia de actuación en dos frentes fundamentales: mejorar las políticas relativas a la sociedad de la información y de I+D y modernizar el modelo social europeo mediante la inversión en capital humano y la lucha contra la exclusión social.

El papel de los sistemas educativos y formativos, en este nuevo modelo de sociedad, quedó definido en el informe del Consejo de Educación al Consejo de 
Europa, The concrete future objectives of education and training systems ${ }^{2}$, presentado en Bruselas en febrero de 2001. El informe analizaba los retos concretos a los que se enfrentaba la Unión y presentaba, a partir de ellos, tres objetivos de carácter estratégico hacia los que debían dirigirse todos los esfuerzos e iniciativas, a lo largo de la década: mejorar la calidad y eficacia de los sistemas de educación y formación en la Unión Europea; facilitar el acceso a dichos sistemas; y abrirlos a la sociedad y al mundo. Las acciones estratégicas vinculadas a estos objetivos estarían coordinadas mediante un método abierto de coordinación ${ }^{3}$ que permitiría una evaluación comparativa de las distintas iniciativas nacionales, con el fin de difundir buenas prácticas y lograr una mayor convergencia hacia las metas de la Unión.

Para cada uno de los objetivos estratégicos, además, el informe identificaba una serie de objetivos asociados, de los que cabe destacar, por su importancia para este trabajo, los siguientes: mejorar la educación y formación de los docentes y formadores; desarrollar las habilidades y destrezas necesarias para la sociedad del conocimiento, especialmente la lectura, la escritura y el cálculo; garantizar el acceso a las tecnologías de la información y la comunicación a todos los ciudadanos; desarrollar el espíritu emprendedor y la autonomía personal; promover los programas de movilidad para facilitar el intercambio de experiencias culturales y de buenas prácticas; y, por supuesto, fomentar el aprendizaje de lenguas extranjeras para el enriquecimiento personal y para la competitividad en el ámbito europeo y mundial.

Si bien este último objetivo tenía un carácter general, en el sentido de que se dirigía a todos los ciudadanos, la reunión del Consejo de Europa en Barcelona, en marzo de $2002^{4}$, marcó el inicio de todo un conjunto de acciones y políticas encaminadas, de forma específica, a propiciar el adelanto progresivo de la edad en que los escolares deberían comenzar el aprendizaje de lenguas extranjeras. Concretamente, se pedían acciones para mejorar las destrezas básicas y la enseñanza de al menos dos lenguas extranjeras desde edades tempranas, acciones que se recogerían en Detailed work programme on the follow-up of the objectives of Education and training systems in Europe (2002)5. Además de señalar las lenguas como el elemento clave de la diversidad europea y su rol estratégico en la construcción de la sociedad del conocimiento, este documento ponía de manifiesto la necesidad de mejorar los métodos de enseñanza de lenguas extranjeras y la formación del profesorado.

${ }^{2}$ Commission of the European Communities, Brussels 31.01.2001 COM (2001) 59 final, Report from the Commission, The Concrete Future Objectives of Education and training Systems, http:// www.aic.lv/ace/ace_disk/Bologna/contrib/EU/concr_obj.pdf.

${ }^{3}$ Lisbon European Council (23-24 March 2000), Conclusions of the Presidency, Bulletin 27.03.2000, p. 24, http://www.europarl.europa.eu/bulletins/pdf/1s2000en.pdf.

${ }_{4}^{4}$ Barcelona European Council (15-16 March 2002), Presidency Conclusions, http://ec.europa.eu/invest-in-research/pdf/download_en/barcelona_european_council.pdf.

${ }_{5}$ Council Information on the Detailed work programme on the follow-up of the objectives of Education and training systems in Europe (2002/C 142/01), Official Journal of the European Communities 14.6.2002, Objective 3.3- Improving Foreign Language Learning, https://publications.europa. eu/en/publication-detail/-/publication/2d17e529-6c81-4b85-8d19-e9dbe65a93c0. 
Más definitivo, aún, el documento Conclusiones del Consejo de mayo de 2009 sobre un Marco estratégico para la cooperación europea en el ámbito de la educación y la formación (ET 2020) ${ }^{6}$ establecía los objetivos para la década siguiente y recogía las áreas de actuación prioritarias (benchmarks) acordadas para facilitar el seguimiento y el logro de dichos objetivos, y para identificar los retos y desafíos que habría que enfrentar. La educación y la formación en el marco ET 2020, además, debían situarse en el ámbito del aprendizaje permanente (lifelong learning) y abarcar los contextos formal, no formal e informal, y todas las etapas y niveles educativos, desde la educación temprana hasta la educación superior, la formación profesional y el aprendizaje de adultos. Resulta lógico entonces, desde esta perspectiva, que se identificara la educación a edades tempranas como una de las áreas de actuación prioritarias, con un objetivo muy específico: lograr, para el año 2020, que el 95\%, al menos, de los niños con edades comprendidas entre los cuatro años y la edad de comienzo de la escolarización obligatoria se beneficiara de la educación. Se instaba, así, a todos los estados miembros a cooperar para alcanzar este objetivo y a impulsar la investigación, el desarrollo y el intercambio de buenas prácticas.

\section{LA ENSEÑANZA DE LENGUAS EXTRANJERAS A EDADES TEMPRANAS}

Con la firma de los acuerdos de Barcelona 2002 y la publicación del Action Plan 2004-06, las políticas educativas europeas han enfatizado la importancia de adelantar la edad de comienzo del aprendizaje de lenguas extranjeras por los beneficios a largo plazo que puede reportar un aprendizaje temprano: a) aumenta la probabilidad de lograr niveles de competencia similares a los de los hablantes nativos, especialmente en la pronunciación y entonación de la lengua (Snow y Hoefnagel-Höhle, 1982a; Asher y García, 1982; Seliger, Krashen y Ladefoged, 1982; Krashen, Long y Scarcella, 1982); b) favorece el desarrollo de la conciencia metalingüística y, por ende, del plurilingüismo (Nikolov y Djigunovic, 2006; Haznedar, 2015; Malkina, 2008), así como los procesos de comprensión, expresión, comunicación y resolución de problemas (Edelenbos, Johnstone y Kubanek, 2006); c) incrementa la concentración, la motivación y la autoconfianza (Kersten, 2015; Enever, 2012); d) facilita la toma de conciencia de la diversidad lingüística y cultural circundante y promueve el diálogo intercultural (Coltrane, 2003; Nikolov y Djigunovic, 2006); e) aunque el aprendizaje infantil es lento, constituye una condición indispensable para que se produzca un aprendizaje eficiente posterior (Ekstrand, 1982); y f) promueve la transferencia de conocimientos y habilidades con la lengua materna (Kersten, 2015; Haznedar, 2015).

${ }^{6}$ Council conclusions of 12 May 2009 on a Strategic Framework for European cooperation in education and training ('ET2020') (2009/C 119/02), http://www.cedefop.europa.eu/files/education_benchmarks_2020.pdf. 
Los beneficios mencionados, cabe señalar, no son intrínsecos a la edad, ni se considera esta ya, por sí sola, un factor determinante del aprendizaje (Johnstone, 2002; Haznedar, 2015). Si bien los resultados de la investigación en lingüística aplicada coinciden ampliamente en que la edad juega un papel fundamental en la adquisición de segundas lenguas y lenguas extranjeras, también convienen en la importancia capital de aspectos tales como el uso de metodologías adecuadas a la edad de los aprendices, las oportunidades de interacción en contextos significativos, la cantidad y calidad del input que se reciba, la motivación del aprendiz y, por supuesto, la formación del profesorado, factor que se percibe como uno de los más importantes, si no el que más, para que el proceso de aprendizaje resulte eficaz y duradero, como se explica a continuación.

Conforme a los Principios comunes europeos para las competencias y cualificaciones del profesorado, establecidos por la Comisión Europea para el ámbito comunitario ${ }^{7}$, la profesión docente debe configurarse como una profesión atractiva y bien cualificada, científica y pedagógicamente; debe situarse, además, en el contexto del aprendizaje y la formación permanentes, transmitir los valores sociales y culturales que conforman la nueva identidad europea a las futuras generaciones de ciudadanos, favorecer la colaboración con el entorno social y potenciar la investigación educativa necesaria para la innovación docente.

El adelanto de la edad para el aprendizaje de lenguas extranjeras, sin embargo, ha puesto de manifiesto una realidad de prácticas muy diferente que describe a docentes generalistas que imparten la lengua meta sin una competencia adecuada, pero con un conocimiento sólido de la etapa; especialistas en la enseñanza de lenguas extranjeras, normalmente de Educación Primaria, que poseen un buen nivel de competencia en la lengua meta, pero carecen de la formación necesaria para enseñarla a estas edades (Nikolov y Djigunovic, 2006; Enever, 2012); profesores nativos con cualificación para impartir la lengua a grupos de edades superiores, o sin cualificación alguna (Copland y Garton, 2014); docentes contratados o itinerantes que imparten solo esta docencia en los centros o, incluso, profesorado con cualificaciones que nada tienen que ver con las lenguas y su enseñanza, en el peor de los casos (Mourao y Ferreirinha, 2016).

Igualmente, el carácter no obligatorio de la etapa y de las lenguas extranjeras a edades tempranas, en algunos países, junto con las presiones y demandas sociales en favor de la introducción de estas - mayoritariamente del inglés-, han dado lugar a una desigualdad de oportunidades y a una variedad de modelos de enseñanza en contextos tanto formales como no formales que incluyen exposición o instrucción formal en una lengua extranjera -language exposure-, Aprendizaje Integrado de Contenidos en Lengua Extranjera -AICLE o CLIL, por sus siglas en inglés-, sensibilización hacia las otras lenguas -language awareness-, docencia compartida

7 Common European Principles for Teacher Competences and Qualifications, European Comission (2005), Directorate General for Education and Culture. http://www.pef.uni-lj.si/bologna/dokumenti/eu-common-principles.pdf. 
en dos lenguas -language tandems-o inmersión bilingüe, por citar algunos (European Commission, 2011b; Edelenbos et al., 2006). Estos modelos, a su vez, determinan no solamente el número y duración de las sesiones - que pueden variar desde una semanal de 30-45 minutos (Mourao y Ferreirinha, 2016) hasta ocho horassino también las metodologías y los medios y materiales a utilizar, por lo general más variados en los contextos no formales (Enever, 2012; Copland y Garton, 2014).

La Clasificación Internacional Normalizada de la Educación (CINE) ${ }^{8}$ (UNESCO, 2013), por su parte, define la «educación en la primera infancia» (p. 28), correspondiente a los programas de Nivel CINE 0, como una etapa de carácter holístico, enfocada al desarrollo cognitivo, físico, social y emocional de los niños, a la relación con la familia y la comunidad y al tránsito a la educación obligatoria. Si bien en este nivel los programas no son altamente estructurados, los entornos de aprendizaje deben ser muy visuales y proporcionar abundante estímulo lingüístico que promueva la adquisición y uso de la lengua en contextos de comunicación significativos, y el desarrollo de las destrezas motoras y de coordinación a través del juego, las actividades lúdicas y la experimentación, como medios para la interacción social con otros nińos y adultos, y para el descubrimiento del mundo que los rodea.

Si el aprendizaje de lenguas extranjeras en la sociedad de la información y el conocimiento debe ser una parte integrante de la educación a edades tempranas, como se ha señalado, resulta evidente, entonces, la necesidad de formar profesorado competente no solo desde el punto de vista lingüístico sino, también, del conocimiento de las características del aprendizaje a estas edades, del currículo y las especificidades de la etapa, y de las metodologías para la enseñanza de lenguas extranjeras que se consideren más apropiadas (Coltrane, 2003; Copland y Garton, 2014; Lungu, 2015). En cualquier caso, la enseñanza de lenguas extranjeras a edades tempranas debe enfocarse hacia las metas siguientes (European Commission, 2011b):

1. Promover la educación intercultural. Puesto que la diversidad lingüística conlleva diversidad cultural, sensibilizar a los niños acerca de este hecho ayuda a prevenir actitudes xenófobas y racistas hacia otras lenguas y culturas y fomenta valores como el respeto, la tolerancia, el diálogo intercultural y la aceptación de la diversidad (Mourao y Lourenço, 2015). En este sentido, establecer relaciones constructivas entre la escuela, la familia y el entorno resulta fundamental (Coltrane, 2003), al ser los ámbitos en los que tiene lugar el primer contacto de los niños con la educación.

2. Fomentar el desarrollo personal de los niños. Las actividades orientadas a sensibilizar acerca de la diversidad lingüística-language awareness-, al igual que las centradas en el aprendizaje de una lengua extranjera concreta-language

${ }^{8}$ La Clasificación Internacional Normalizada de la Educación (CINE), aprobada por la 36. ${ }^{a}$ Conferencia General de la UNESCO en 2011, constituye un marco de referencia que permite ordenar los programas educativos y sus respectivas certificaciones por niveles de educación y campos de estudio. "Las definiciones y conceptos básicos de la CINE se han formulado de modo que sean universalmente válidos y aplicables al espectro total de sistemas educativos» (UNESCO, 2013, p. 6). 
exposure-, contribuyen al desarrollo global de las competencias y destrezas de los nińos, por lo que constituyen una oportunidad de desarrollo social, emocional y cognitivo (Lourenço y Andrade, 2015). Estas actividades, además, deben tener en cuenta sus intereses y necesidades y facilitar el paso a Primaria.

3. Favorecer el aprendizaje permanente. Una enseñanza de lenguas eficaz implica, necesariamente, un compromiso de continuidad respecto a la opción de lengua que se aprende inicialmente y a los métodos y enfoques con los que se imparte, en el tránsito entre etapas (Nikolov y Djigunovic, 2006). Los programas de lenguas deben adaptarse a cada contexto particular, partir del conocimiento previo de los niños y despertar su interés, motivación y curiosidad hacia lenguas y culturas diferentes de la propia. El contacto con los sonidos y la entonación de las lenguas son particularmente importantes en esta etapa.

Desde el punto de vista pedagógico didáctico, además:

1. La lengua extranjera debe enseñarse de forma holística, atendiendo al principio de globalización o integración de contenidos que caracteriza a la etapa, y no como un contenido extra, es decir, debe contribuir a reforzar el desarrollo y las capacidades de los niños, mediante actividades significativas que respondan a sus intereses y a las necesidades de su entorno inmediato (Edelenbos, Johnstone y Kubanek, 2006; European Commission, 2011b). En los contextos en los que la lengua no sea impartida por generalistas con la formación necesaria para ello, se recomienda la docencia compartida con los especialistas, que implica no solo la presencia de ambos en el aula sino, también, la adecuada planificación, implementación y evaluación del aprendizaje (Mourao y Ferreirinha, 2016).

2. Se debe tener en cuenta, igualmente, el principio fundamental de proporcionar input que resulte comprensible y ajustado a estas edades, mediante elementos no solo verbales, sino gestuales y visuales (Krashen, 1986), así como actividades que impliquen una respuesta física (Asher, 1988) para asegurar los procesos de comprensión, requisito indispensable para los de producción, y el uso de prácticas andamiadas que faciliten el descubrimiento y el progreso en el aprendizaje (Wood, Bruner y Ross, 1976).

3. Asimismo, la lengua debe enseñarse como un instrumento para la comunicación y para la resolución de otras actividades, en contextos cotidianos y significativos para los niños, y no como una asignatura más. Se recomienda, especialmente, el modelo de aprendizaje basado en tareas y proyectos, el uso de materiales reales y la introducción a las tecnologías de la información y la comunicación (Edelenbos, Johnstone y Kubanek, 2006; Mourao y Lourenço, 2015; European Commission, 2011b).

4. Teniendo en cuenta lo señalado anteriormente, las actividades más apropiadas son, entonces, aquellas con marcado carácter lúdico y que implican experimentación y descubrimiento, uso de elementos visuales y gestuales, además 
de la lengua y actividad física, tales como juegos, canciones, cuentacuentos, marionetas, dramatizaciones, role plays, películas infantiles, uso de internet, actividades plásticas y manualidades, etc. (Malkina, 2008; Enever, 2012; Copland y Garton, 2014; Mourao y Ferreirinha, 2016).

Contrariamente a la creencia, tan común y errónea, acerca de la formación y la cualificación del profesorado necesarias para enseñar lenguas extranjeras a estas edades, la realidad ha demostrado que cuanto más jóvenes son los aprendices, más necesario resulta contar con profesorado cualificado. Algunos investigadores, de hecho, han recomendado el nivel C1 del Marco Común Europeo de Referencia para las Lenguas (Cerná, 2015) como referente para la competencia lingüística, si bien la cualificación de los docentes, como se ha señalado, debe abarcar todas las áreas que inciden en la educación a edades tempranas.

\section{DESCRIPCIÓN DEL ESTUDIO}

La finalidad de este estudio consistió en identificar las creencias de dos grupos de estudiantes de tercer curso del Grado de Maestro de Educación Infantil acerca de la necesidad de formar profesorado competente para enseñar lenguas extranjeras -inglés y francés- en esta etapa. Los datos para el estudio se recogieron mediante un cuestionario que se administró en la asignatura Didáctica de la lengua extranjera, que se imparte en la Facultad de Educación de la Universidad de La Laguna.

\subsection{Contexto}

El Grado de Maestro de Educación Infantil comprende un plan de estudios de 240 créditos, distribuidos a lo largo de cuatro cursos. La formación en lengua extranjera se organiza en dos asignaturas, Lengua extranjera y Didáctica de la lengua extranjera, ambas con carácter obligatorio y seis créditos de duración, que se cursan en las modalidades de inglés o francés. Estas asignaturas se sitúan en el segundo y tercer año, respectivamente, y constituyen la única formación en una lengua extranjera y su didáctica que recibe un futuro egresado con perfil generalista, $o$ con alguna de las menciones que se ofertan en cuarto curso: Mención en atención a la diversidad, Mención en atención temprana y Mención en animación a la lectura y formación de lectores.

Resulta conveniente señalar que la diferenciación de perfiles mencionada se justifica por la elección de optativas que se realice, y que debe sumar un total de 18 créditos. Un título con mención implica realizar esos 18 créditos de optativas vinculadas a una misma mención y un practicum específico de 12 . Sin embargo, si se opta por cursar los 18 créditos de optativas de menciones diferentes, los 12 de prácticas se cursan como parte del practicum generalista, y el perfil final no comporta mención. Un $87,7 \%$ del total del alumnado matriculado este curso optó por alguna de esas menciones. 
Por último, solo aquellos que cursan la Mención en lengua extranjera, que se oferta únicamente en la modalidad de inglés, reciben más formación en la lengua y su didáctica, con tres asignaturas de carácter optativo y seis créditos cada una: Idioma Extranjero, Diseño y Aplicación Práctica de Unidades de Trabajo en la Lengua Extranjera y El uso de las TIC en la Enseńanza de la Lengua Extranjera, y doce créditos de Practicum de Mención. Es importante destacar, sin embargo, que esta mención se imparte de manera conjunta en Infantil y Primaria, por lo que los alumnos del título que nos ocupa no reciben una formación específica para la etapa, sino un planteamiento más propio de la siguiente, un error evidente que se repite, también, en otros países comunitarios (European Commission, 2011b). El porcentaje de alumnos de Infantil que cursó esta mención, en el presente curso, es de un $12,3 \%$, frente al $46,2 \%$ del alumnado de Primaria ( $43 \%$ en inglés y $3,2 \%$ en francés).

\subsection{Participantes}

El número de estudiantes matriculados en la asignatura Didáctica de la lengua extranjera (inglés), durante el curso académico 2018-2019, sumó un total de 104, de los cuales 89 asistían regularmente a clase. De estos, 84 respondieron al cuestionario, que se administró a principio de curso para no influenciar sus respuestas, quedando la muestra final compuesta por 78 mujeres y seis hombres, con edades comprendidas entre los 22 y los 26 años de edad.

En cuanto al segundo grupo, de las 13 mujeres matriculadas en Didáctica de la lengua extranjera (francés), solamente las nueve que asistían con regularidad, en el mismo rango de edades del grupo anterior, respondieron al cuestionario. Es necesario aclarar que la disparidad evidente en el número de participantes en cada muestra no hizo posible realizar un estudio comparativo, como se pretendía inicialmente; sin embargo, el valor y el interés de los datos obtenidos justifican, sobradamente, su inclusión y análisis en esta investigación.

\subsection{INSTRUMENTO PARA LA RECOGIDA DE DATOS}

La recogida de los datos se realizó mediante un cuestionario de 13 ítems ${ }^{9}$ al que los informantes debían responder conforme a las cinco opciones de la escala Likert: 1 (muy de acuerdo), 2 (de acuerdo), 3 (ni de acuerdo ni en desacuerdo/neutral), 4 (en desacuerdo), 5 (muy en desacuerdo). Para la elaboración del cuestionario se utilizó, parcialmente, el modelo original de Fojkar (2016), al que se añadieron ítems de elaboración propia que reflejan las necesidades de formación del profesorado de lenguas extranjeras a edades tempranas, conforme se identifican en el documento Language learning at pre-primary school level: making it efficient and sustaina-

\footnotetext{
${ }^{9}$ En el anexo 1 se ofrece traducción propia de los ítems del cuestionario.
} 
ble. A policy handbook (European Commission, 2011b), usado en esta investigación. Para facilitar el análisis de los datos, los 13 ítems se agruparon en cuatro áreas de competencia -lingüística, pedagógica, profesional e intercultural-que reflejan, de manera precisa, las necesidades formativas mencionadas.

\subsection{AnÁlisis de los DATOS y Resultados}

Para el análisis de los datos se utilizó el paquete estadístico SPSS versión 25 y una descripción de frecuencias. Las tablas que se incluyen a continuación muestran las cinco opciones de la escala Likert colapsadas en tres. Se presentan, en primer lugar, las frecuencias de la muestra de inglés y, seguidamente, las de francés.

\begin{tabular}{|c|c|c|c|}
\hline \multicolumn{4}{|c|}{ 1. TABLA DE FRECUENCIAS SEGÚN COMPETENCIA LINGÜÍSTICA } \\
\hline Statements & $1 / 2$ & 3 & $4 / 5$ \\
\hline $\begin{array}{l}\text { 8. My competence in the foreign language allows me to understand, speak, read } \\
\text { and write the foreign language fluently, without any problems. }\end{array}$ & $44 \%$ & $31 \%$ & $25 \%$ \\
\hline 11. I am competent enough in the foreign language to teach preschool children. & $29,8 \%$ & $34,5 \%$ & $35,7 \%$ \\
\hline
\end{tabular}

1. Strongly agree; 2. Agree; 3. Neither agree nor disagree, (Neutral); 4. Disagree; 5 . Strongly disagree.

Conforme a los datos obtenidos, el $44 \%$ de los informantes afirman que su competencia en la lengua extranjera les permite poner en práctica las destrezas básicas sin problemas, frente al $56 \%$ restante, que muestran una opinión neutral $(31 \%)$ o en desacuerdo (25\%). Estos datos podrían resultar un tanto contradictorios si consideramos que el $70,2 \%$ de respuestas al ítem siguiente apuntan que esa competencia en la lengua no es suficiente para enseñarla a edades tempranas, frente a solo un $29,8 \%$ que creen que sí lo es. Casi un tercio (14,2\%) de los que opinaban que su competencia era suficiente se decantan, ahora, por una respuesta neutral o en desacuerdo, principalmente. Este dato podría indicar que los informantes tienen una percepción diferente de lo que implica ser competentes como usuarios y como docentes, en cuyo caso estarían mostrando conciencia de que enseñar una lengua requiere algo más que solo competencia en la misma. Eso, al menos, es lo que parece desprenderse de las respuestas a las áreas de competencia siguientes.

\begin{tabular}{|c|c|c|c|}
\hline Statements & $1 / 2$ & 3 & $4 / 5$ \\
\hline 1. It is easier for children than adults to learn a foreign language. & $88,1 \%$ & $8,3 \%$ & $3,6 \%$ \\
\hline $\begin{array}{l}\text { 3. Early foreign language learning may have a negative impact on first language } \\
\text { acquisition. }\end{array}$ & $15,5 \%$ & $22,6 \%$ & $61,9 \%$ \\
\hline $\begin{array}{l}\text { 4. The earlier a foreign language is introduced in school programs, the greater } \\
\text { the likelihood of success in learning. }\end{array}$ & $65,1 \%$ & $27,7 \%$ & $7,2 \%$ \\
\hline
\end{tabular}


5. Early foreign language teaching necessarily demands knowledge of both the foreign language and of the preschool curriculum and teaching methodology.

$75,4 \% \quad 23,5 \%$ $1,2 \%$

6. An inappropriate approach in early foreign language teaching may have a negative effect on children's foreign language learning and motivation.

$89 \%$

$3,7 \%$

$7,3 \%$

7. It is not necessary to have a good command of the foreign language to teach preschool children because their language needs are very basic.

$16,6 \% \quad 17,9 \%$

$65,5 \%$

1. Strongly agree; 2. Agree; 3 . Neither agree nor disagree, (Neutral); 4. Disagree; 5. Strongly disagree.

La mayor parte de los informantes $(88,1 \%)$, con diferencia, está de acuerdo con la afirmación de que los niños aprenden una lengua extranjera más fácilmente que los adultos, que el aprendizaje de lenguas a edades tempranas no tiene un impacto negativo en la adquisición de la materna $(61,9 \%)$ y que cuanto antes comiencen su aprendizaje en la escuela, mayores serán las posibilidades de éxito $(65,1 \%)$. Aunque la investigación llevada a cabo en lingüística aplicada desde la década de los ochenta ha demostrado, ampliamente, que los niños no adquieren una segunda lengua más rápidamente ni mejor que los adultos a corto plazo, excepto la pronunciación y entonación, esta idea sigue siendo muy popular debido, muy probablemente, a la observación de situaciones cotidianas, carentes de base científica (Asher y Ramíez, 1982).

A largo plazo, sin embargo, sí muestran una competencia mayor, que no interfiere negativamente en el desarrollo de la lengua materna (Coltrane, 2003), si bien puede haber transferencia léxica (Snow y Hoefnagel-Höhle, 1982b). Esta diferencia de competencia, que suele darse al cabo de un año, puede deberse a factores tales como la continuidad en el proceso de adquisición, la necesidad de asegurar la comunicación, su motivación integradora (Snow y Hoefnagel-Höhle, 1982a) o el tipo de input que reciben (Scarcella y Higa, 1982). En los contextos de aprendizaje de lenguas extranjeras, específicamente, estos factores, sumados a otros ya señalados como las metodologías de enseñanza, el tiempo de exposición a la lengua o la formación del profesorado responsable de su impartición, son los que, decididamente, pueden determinar el éxito del aprendizaje a edades tempranas (Nikolov y Djigunovic, 2006; Haznedar, 2015).

Más interesante, incluso, resulta que el 75,4\% están de acuerdo o muy de acuerdo en que la enseñanza de lenguas extranjeras demanda no solo conocimiento de la lengua, sino de las metodologías y el currículo específicos de la etapa, y que un enfoque inadecuado podría causar efectos negativos en el aprendizaje y la motivación (89\%). Igualmente, el $65,5 \%$ rechazan la afirmación de que no es necesario contar con una buena competencia en la lengua para enseñarla a los nińos porque sus necesidades comunicativas son muy básicas. Las respuestas a los tres ítems, como se puede observar, están totalmente en sintonía con las recomendaciones de la Comisión Europea y la investigación citada en este estudio. Además, dan consistencia al argumento en favor de una percepción de la enseñanza a edades tempranas que requiere más formación que solo el conocimiento de la lengua, como ya se apuntó con respecto a los datos de la competencia lingüística. 


\section{TABLA DE FRECUENCIAS SEGÚN COMPETENCIA PROFESIONAL}

\begin{tabular}{lccc}
\hline \multicolumn{1}{c}{ STAтEMENTs } & $1 / 2$ & 3 & $4 / 5$ \\
\hline $\begin{array}{l}\text { 9. I would like to teach the foreign language to preschool children in my future } \\
\text { teaching career. }\end{array}$ & $62 \%$ & $21,3 \%$ & $16,7 \%$ \\
\hline $\begin{array}{l}\text { 10. I would not mind taking up courses in the foreign language and foreign } \\
\text { language teaching methodology to teach preschool children in the future. }\end{array}$ & $74,3 \%$ & $17,1 \%$ & $8,6 \%$ \\
\hline $\begin{array}{l}\text { 12. It is very unlikely that preschool teachers will have to teach the foreign } \\
\text { language to preschoolers. }\end{array}$ & $10,8 \%$ & $20,5 \%$ & $68,7 \%$ \\
\hline $\begin{array}{l}\text { 13. Preschool teachers should not be responsible for teaching the foreign language } \\
\text { to preschoolers because it is not their specialism. }\end{array}$ & $12 \%$ & $14,5 \%$ & $73,5 \%$ \\
\hline
\end{tabular}

1. Strongly agree; 2. Agree; 3. Neither agree nor disagree, (Neutral); 4. Disagree; 5. Strongly disagree.

En cuanto a la competencia profesional, el $62 \%$ de los informantes afirman que les gustaría enseñar la lengua extranjera en esta etapa y que, además, estarían dispuestos a realizar cursos de perfeccionamiento en la lengua y en la metodología para su enseñanza, en el futuro (74,3\%). Estas afirmaciones son coherentes con las respuestas a los dos ítems siguientes, ya que el $68,7 \%$ no creen improbable que los docentes de esta etapa tengan que enseñar la lengua a los nińos, aunque no sea su especialidad $(73,5 \%)$. Es necesario destacar la importancia de estos datos ya que, si se considera que, en las anteriores diplomaturas de maestro, el título de Infantil era el único de los cinco ofertados que no incluía créditos de lengua extranjera, y que esta idea ha seguido presente en la mente de muchos alumnos, parece obvio, ahora, que se está creando una conciencia más clara acerca de la importancia de las lenguas en la etapa y, por supuesto, de la empleabilidad que ello comporta.

\begin{tabular}{|c|c|c|c|}
\hline \multicolumn{4}{|c|}{ 4. TABLA DE FRECUENCIAS SEGÚN COMPETENCIA INTERCULTURAL } \\
\hline Statements & $1 / 2$ & 3 & $4 / 5$ \\
\hline $\begin{array}{l}\text { 2. Early foreign language learning helps children learn about other cultures and } \\
\text { accept them. }\end{array}$ & $85,5 \%$ & $10,8 \%$ & $3,6 \%$ \\
\hline
\end{tabular}

1. Strongly agree; 2. Agree; 3. Neither agree nor disagree, (Neutral); 4. Disagree; 5. Strongly disagree.

Por último, la importancia de sensibilizar a los más pequeńos acerca de las diferencias culturales resulta obvia, ya que un destacado $85,5 \%$ así lo entienden. La presencia de niños con lenguas y culturas diferentes a las propias es un hecho cada vez más cotidiano y, si bien esta diversidad es más evidente en zonas concretas, no resulta, en absoluto, infrecuente contar con menores de procedencia diversa que se integran, sin dificultades a estas edades, en las aulas. Este es un hecho constatado por los propios alumnos del Grado durante los periodos de prácticas externas en los centros, prácticas que contribuyen a su comprensión de la realidad educativa actual:

Los valores que han de estar presentes a lo largo de toda la vida educativa son el respeto a los principios democráticos, a los derechos y deberes ciudadanos y a las libertades fundamentales, y todos aquellos que cumplan las exigencias individuales 
de una vida en común, educando en la sensibilidad y en la tolerancia. La escuela se configura como contexto donde se construyen culturas y se crean espacios de compensación de diferencias asociadas a factores de origen económico, cultural y social. $\left(B O C\right.$ n. ${ }^{\circ} 163,2008$, p. 15979) ${ }^{10}$.

\section{TABLA 5. FRECUENCIAS DE LA MUESTRA DE FRANCÉS: COMPETENCIAS LINGÜÍSTICA Y PEDAGÓGICA}

\begin{tabular}{llll}
\hline Frecuencias SEgún COMPETENCIA LINGÜÍstica & $1 / 2$ & 3 & $4 / 5$ \\
\hline
\end{tabular}

8. Ma compétence en langue étrangère me permet de comprendre, de parler, de lire et d'écrire cette langue étrangère couramment, sans aucun problème.

$22,2 \% \quad 55,6 \% \quad 22,2 \%$

11. Je suis assez compétent dans la langue étrangère cible pour enseigner aux enfants d'âge préscolaire.

$33,3 \% \quad 33,3 \% \quad 33,3 \%$

Frecuencias SEgún COMPETENCIA PEDAGógica $\quad 1 / 2 \quad 3 \quad 4 / 5$

1. Il est plus facile pour les enfants que pour les adultes d'apprendre une langue étrangère.

$77,7 \%$

$22,2 \%$

3. L'apprentissage précoce des langues étrangères peut avoir un impact négatif sur l'acquisition d'une langue première.

4. Plus tôt une langue étrangère est introduite dans les programmes scolaires, plus grande est la probabilité de succès dans son apprentissage.

$66,6 \% \quad 33,3 \%$

5. L'enseignement précoce des langues étrangères exige à la fois, la maîtrise de la langue étrangère et la connaissance du programme d'enseignement et de la $\quad 11,1 \% \quad 44,4 \% \quad 44,4 \%$ méthodologie d'enseignement préscolaire.

6. Une approche inappropriée dans l'enseignement précoce des langues étrangères peut avoir un effet négatif sur l'apprentissage des langues étrangères et sur la motivation des enfants.

7. Il n'est pas nécessaire d'avoir une bonne maîtrise de la langue étrangère pour enseigner aux enfants d'âge préscolaire parce que leurs besoins linguistiques sont très élémentaires.

$11,1 \% \quad 22,2 \% \quad 66,6 \%$

1. Tout à fait d'accord; 2. D'accord; 3. Sans avis; 4. Pas d'accord; 5. Pas du tout d'accord.

Con respecto a la muestra de francés, solo el $22,2 \%$ de los alumnos responden de forma positiva respecto a su competencia para comprender y producir verbalmente y por escrito la lengua extranjera, frente al $77,8 \%$ con una opinión en desacuerdo $(22,2 \%)$ o neutral $(55,6 \%)$. Esta tendencia se repite con respecto a la competencia para enseńarla, con un 33,3\% de acuerdo y un 66,6\% en desacuerdo $(33,3 \%)$ y neutrales $(33,3 \%)$. Se observa, por tanto, una disminución del $22,2 \%$ de

${ }^{10}$ Consejería de Educación, Universidades y Deportes (2008), Decreto 183/2008, de 29 de julio, por el que se establece la ordenación y el currículo del $2 .^{\circ}$ ciclo de la Educación Infantil en la Comunidad Autónoma de Canarias, Boletín Oficial de Canarias núm. 163, jueves 14 de agosto de 2008. 
respuestas neutrales respecto al ítem 1, en favor de los otros dos grupos, que aumentan ahora en un $11,1 \%$.

En cuanto a la competencia pedagógica, más de la mitad de los informantes se muestran de acuerdo en que los niños aprenden una lengua extranjera más fácilmente que los adultos (77,7\%), y que su aprendizaje a edades tempranas no tiene un impacto negativo en la adquisición de la lengua materna $(88,8 \%)$. Dos tercios $(66,6 \%)$, además, opinan que las probabilidades de éxito en el aprendizaje aumentan con la escolarización temprana, frente a un tercio que se mantiene neutral.

Por otro lado, resulta contradictorio, y curioso a la vez, que el 88,8\% se manifiesten en desacuerdo $(44,4 \%)$ o neutral $(44,4 \%)$ acerca de la necesidad de conocer la lengua extranjera y las metodologías y el currículo específicos de la etapa para enseñarla, pero acepten, mayoritariamente $(88,9 \%)$, que un enfoque inadecuado podría causar efectos negativos en el aprendizaje y la motivación, como si esta última fuera solo intrínseca al aprendiz, o no estuviera vinculada a los aspectos didáctico-pedagógicos. La respuesta al ítem siguiente resulta más llamativa aún, ya que el 66,6\% reconocen que es necesario contar con una buena competencia en la lengua extranjera para enseñarla, aunque las necesidades comunicativas de los nińos sean muy básicas, si bien las respuestas a los ítems relacionados con la competencia lingüística no parecían evidenciarlo.

\begin{tabular}{|c|c|c|c|}
\hline \multicolumn{4}{|c|}{$\begin{array}{l}\text { TABLA 6. FRECUENCIAS DE LA MUESTRA DE FRANCÉS: } \\
\text { COMPETENCIAS PROFESIONAL E INTERCULTURAL }\end{array}$} \\
\hline FRECUENCIAS SEGÚN COMPETENCIA PROFESIONAL & $1 / 2$ & 3 & $4 / 5$ \\
\hline $\begin{array}{l}\text { 9. Je voudrais enseigner cette langue étrangère aux enfants d'âge préscolaire dans } \\
\text { ma future carrière d'enseignant. }\end{array}$ & $66,6 \%$ & $22,2 \%$ & $11,1 \%$ \\
\hline $\begin{array}{l}\text { 10. Ça ne me dérangerait pas de prendre des cours de langue étrangère et de } \\
\text { méthodologie d'enseignement des langues étrangères pour enseigner aux enfants } \\
\text { d'âge préscolaire à l'avenir. }\end{array}$ & $55,5 \%$ & $22,2 \%$ & $22,2 \%$ \\
\hline $\begin{array}{l}\text { 12. Il est très peu probable que les enseignants du préscolaire doivent un jour } \\
\text { enseigner une langue étrangère aux enfants d'âge préscolaire. }\end{array}$ & $44,4 \%$ & $11,1 \%$ & $44,4 \%$ \\
\hline $\begin{array}{l}\text { 13. Les enseignants du préscolaire ne devraient pas être responsables de l'ensei- } \\
\text { gnement d'une langue étrangère aux enfants d'âge préscolaire, car ce n'est pas } \\
\text { leur spécialité. }\end{array}$ & $22,2 \%$ & $22,2 \%$ & $55,5 \%$ \\
\hline FRECUENCIAS SEGÚN COMPETENCIA INTERCULTURAL & $1 / 2$ & 2 & $4 / 5$ \\
\hline $\begin{array}{l}\text { 2. L'apprentissage précoce des langues étrangères aide les enfants à apprendre } \\
\text { d'autres cultures et à les accepter. }\end{array}$ & $100 \%$ & - & - \\
\hline
\end{tabular}

Por otro lado, si bien una parte importante de informantes reconocieron no tener competencia suficiente en la lengua, o respondieron de forma neutral al respecto, dos tercios $(66,6 \%)$ se muestran, ahora, favorables a enseñarla en el futuro, y más de la mitad del total $(55,5 \%)$ estarían dispuestos a formarse para ello. El 
mismo porcentaje $(44,4 \%)$ aceptan y descartan la posibilidad de que los docentes de esta etapa impartan la lengua extranjera, pero más de la mitad $(55,5 \%)$ opinan que pueden hacerlo, aunque no sea su especialidad. Esta incertidumbre acerca de la enseñanza del francés se justifica, razonablemente, si se tiene en cuenta, como ya se explicó, que el inglés es, de momento, la única lengua extranjera impartida en los centros escolares. Todos (100\%), sin embargo, están convencidos de los beneficios que comporta el contacto con otras las lenguas y sus culturas desde edades tempranas.

\section{CONCLUSIONES DEL ESTUDIO}

La finalidad de este estudio consistió en identificar las creencias de dos grupos de alumnos de tercer curso del Grado de Maestro de Educación Infantil acerca de la necesidad de formar profesorado competente para enseñar lenguas extranjeras -inglés y francés- en la etapa. Los resultados de ambas muestras indican una actitud positiva y optimista no solo respecto a la formación de los docentes, sino al contacto de los escolares con estas lenguas y sus culturas a edades tempranas.

Si bien es cierto que solo el $44 \%$ de los alumnos de inglés se consideran competentes en la lengua, más de la mitad admiten que les gustaría dedicarse profesionalmente a su enseñanza y que estarían dispuestos a recibir cursos específicos, como parte de su formación futura, ya que entienden que las necesidades formativas de esta etapa demandan mucho más que competencia lingüística. Por ello, justamente, rechazan que sean otros los idóneos para enseñarla y que, si bien no es su especialidad, ciertamente poseen suficiente conocimiento del currículo y de las características de la etapa, como parte de su formación en el Grado. Todos ellos, además, reconocen los beneficios que comporta el contacto con otras lenguas y culturas en la construcción de valores y en la aceptación de la diversidad.

Estas mismas conclusiones son válidas respecto al grupo de alumnas de francés, que, aunque muestran menos competencia en la lengua y carecen de una idea bien definida acerca de la formación que demanda su enseñanza en la etapa, reconocen los beneficios de un contacto temprano y se manifiestan, igualmente, abiertas a recibir formación específica para el ejercicio profesional. Todo ello, además, en un contexto en que el francés no forma parte, aún, de la oferta docente de la Educación Infantil.

Si se consideran las directrices europeas respecto al papel de las lenguas extranjeras en la construcción de la sociedad de la información y el conocimiento y en las opciones de empleabilidad, y se atiende a la conveniencia de adelantar su aprendizaje a edades tempranas, como ya se puso de manifiesto en la Resolución del Consejo de la Unión Europea de 1997, contar con profesorado cualificado para atender, específicamente, a las necesidades de aprendizaje de lenguas de los más pequeños resulta, en el contexto al que se refiere esta investigación, una prioridad evidente para los alumnos del Grado que, paradójicamente, no se refleja en la nueva configuración del título aprobada por los profesionales y equipos directivos responsables de la toma de decisiones. En la sociedad de la información y el conocimiento, el papel de las lenguas extranjeras resulta crítico y, por ello, es un derecho de los ciu- 
dadanos recibir esa formación, y una obligación de los responsables ofrecerla en las mejores condiciones posibles.

Por otro lado, el planteamiento de la Consejería de Educación respecto a la enseñanza del inglés como única lengua extranjera en Educación Infantil, en función, además, de la disponibilidad horaria de los actuales especialistas de Educación Primaria, que, en muchos casos, ni tan siquiera siguen el enfoque globalizador recomendado para la etapa, y la perspectiva de perpetuarlo, a juzgar por las medidas que se han ido implementado desde la administración educativa autonómica y desde la propia universidad, esta última con una "política lingüística» estrecha y contraria a las propias recomendaciones europeas en materia de lenguas, ponen de manifiesto una clara falta de previsión y aprovechamiento de las condiciones favorables de nuestro sistema educativo y del capital humano disponible en nuestro contexto para promover acciones de iniciación y sensibilización de los escolares hacia el aprendizaje de dos lenguas extranjeras y sus culturas.

Sería necesario, por tanto, poner en marcha medidas y actuaciones en línea con las recomendaciones y directrices de la Unión Europea, ajenas al devenir de los tiempos políticos y de circunstancias externas a las propias necesidades formativas de nuestro alumnado y de los escolares de la etapa para la que se forman. Esto implicaría, en su vertiente más altruista y filosófica, la necesidad de concienciar a la sociedad, en general, y a la comunidad educativa, en particular, de las ventajas que suponen las lenguas extranjeras para el desempeño personal y profesional, en el modelo social europeo en el que estamos inmersos, y la de comprender el valor de las lenguas extranjeras como instrumento para la comunicación y el diálogo intercultural; en definitiva, una acción coordinada de todos los agentes sociales que intervienen en la educación de nuestros escolares y en la formación de sus futuros docentes.

Por otro lado, requeriría la puesta en marcha, por parte de la administración autonómica, de proyectos piloto de introducción de la segunda lengua extranjera -francés- en Educación Infantil, tal como hizo, en su día, respecto al inglés, garantizando, igualmente, su continuidad en la etapa siguiente, Primaria; para ambas lenguas, además, sería fundamental actualizar y adecuar el orden de prioridad de impartición a las circunstancias presentes, y establecer directrices claras y precisas respecto a la certificación de nivel de lengua requerido, conforme al Marco Común Europeo de Referencia para las Lenguas. Estas medidas, sin duda, contribuirían a dar sentido y prestigiar las menciones que se vinculen al Grado de Infantil y, también, a las que conserva Primaria.

Obviamente, cualquier medida que se tome debe ir respaldada por una apuesta formativa clara y coherente que contemple acciones dirigidas a mejorar, como mínimo, los planes de estudio, las infraestructuras y los recursos y medios materiales disponibles, las oportunidades de formación continua del profesorado universitario y los sistemas de garantía de calidad del título. Definir un perfil claro del docente de lenguas extranjeras adecuado a las necesidades formativas de la etapa; reducir la ratio de alumnado por grupo; ampliar la oferta de materias en ambas lenguas y secuenciarlas a lo largo de los cuatro cursos del título, con carácter obligatorio para todo el alumnado; establecer un abanico de optativas que cubra no solo aspectos lingüísticos sino culturales e interculturales; poner a disposición del alum- 
nado medios, materiales y espacios adecuados para facilitar el aprendizaje autónomo y compensar sus carencias lingüísticas, cada vez más evidentes a pesar de las ventajas de las tecnologías de la información y la comunicación y de las posibilidades de viajar a países meta; incentivar las iniciativas de formación con reconocimiento de créditos; importar la figura del profesor ayudante (assistant teacher); facilitar espacios de coordinación entre el profesorado de las distintas lenguas y la docencia compartida; promover programas de seguimiento y evaluación de los planes de estudio enfocados a detectar o diagnosticar debilidades, no a satisfacer meros trámites administrativos, y proponer medidas de subsanación; incentivar la formación permanente del profesorado universitario mediante una oferta formativa atractiva y coherente; promover la movilidad nacional e internacional de profesores y alumnos y el intercambio de buenas prácticas; y, por supuesto, exigir estándares de compromiso, responsabilidad y ética personal y profesional adecuados a la profesión docente son algunas de las medidas y acciones que, sin duda, contribuirían a fomentar el interés por las lenguas extranjeras y sus culturas a edades tempranas, a crear las condiciones idóneas para introducirlas en la etapa y a construir esa sociedad de la información y el conocimiento que, por el momento, parece solo "de papel», en las mismas condiciones que nuestros socios europeos. 


\section{REFERENCIAS}

Asher, J. y García, R. (1982). «The optimal age to learn a foreign language», en S.D. Krashen, R.C. Scarcella y M.H. Long (eds.), Child-Adult differences in second language acquisition (pp. 3-12). Rowley, Massachusetts: Newbury House Publishers, Inc.

Asher, J. (1988). Learning another language through actions. The complete teacher's guidebook. Los Gatos, California: Sky Oaks Productions, Inc.

Cenná, M. (2015). «Pre-primary English language learning and teacher education in the Czeck Republic», en S. Mourao y M. Lourenço (eds.), Early years second language education. International perspectives on theory and practice (pp. 165-176). New York: Routledge.

Coltrane, B. (2003). «Working with young English language learners: some considerations». ERIC Digest. ED48169, ERIC Clearinghouse on Language and Linguistics, Washington D.C., pp. 1-7.

Copland, F. y Garton, S. (2014). «Key themes and future directions in teaching English to young learners». ELT Journal, 68: 223-230. Recuperado de https://academic.oup.com/eltj/articleabstract/68/3/223/45801.

Edelenbos, P., Johnstone, R. y Kubanek, A. (2006). «The main pedagogical principles underlying the teaching of languages to very young learners. Languages for the children of Europe. Published research, good practice \& main principles». European Commission: Final Report of the EAC 89/04, Lot 1 stud. Recuperado de http://ec.europa.eu/assets/eac/languages/policy/ languagepolicy/documents/young_en.pdf.

ENEver, J. (2012). «Current policy issues in early foreign language learning». CEPS Journal, 2: 9-25. Recuperado de https://core.ac.uk/download/pdf/27154422.pdf.

EnEver, J. (2016). "What can we expect of an early start to foreign language learning in Europe today?» Gyermeknevelés, 4: 2-10. Recuperado de http://gyermekneveles.tok.elte.hu/16_1_ szam/pub/enever.pdf.

Ekstrand, L. (1982). «English without a book revisited: the effect of age on second language acquisition in a formal setting", en S.D. Krashen, R.C. Scarcella y M.H. Long (eds.), Child-Adult differences in second language acquisition (pp. 136-158). Rowley, Massachusetts: Newbury House Publishers, Inc.

European Commission (2011b). Language learning at pre-primary school level: making it efficient and sustainable. A policy handbook. (Brussels, 7.7.2011 SEC 928 final). Recuperado de http:// ec.europa.eu/assets/eac/languages/policy/language-policy/documents/early-languagelearning-handbook_eu.pdf.

European Commission (2004). Promoting language learning and linguistic diversity. An action plan 2004-06. Recuperado de http://www.saaic.sk/eu-label/doc/2004-06_en.pdf.

European Council (2000). Lisbon European Council (23-24 March 2000), Conclusions of the Presidency. (Bulletin 27.03.2000). Recuperado de http://www.europarl.europa.eu/bulletins/ pdf/1s2000en.pdf.

FojKar, M.D. (2016). «Pre-primary teachers' beliefs about early foreign language learning in Slovenia». Journal of Foreign Language Teaching and Applied Linguistics, 3: 1-8. DOI: 10.14706/JFLTAL163113.

Gobierno de Canarias (2000). «Orden de 26 de mayo de 2000, por la que se regula la implantación, con carácter experimental, del inglés en el segundo ciclo de la Educación Infantil». (BOC 
núm. 72, lunes 12 de junio). Recuperado de http://www.gobiernodecanarias.org/juriscan/ ficha.jsp?id=23525.

Gobierno de Canarias (2002). «Orden de 20 de mayo de 2002, por la que se regula la implantación de la enseñanza del inglés como primer idioma a partir del segundo ciclo de Educación Infantil en centros docentes de Educación Infantil y Primaria pertenecientes a la Consejería de Educación, Cultura y Deportes de la Comunidad Autónoma de Canarias». (BOC núm. 88 , viernes 28 de junio). Recuperado de http://www.gobiernodecanarias.org/boc/2002/088/ boc-2002-088-006.pdf.

Gobierno de Canarias (2002). "Resolución de 22 de julio de 2002 por la que se dictan las instrucciones para la implantación de la enseñanza del inglés como primera lengua extranjera a partir del segundo ciclo de Educación Infantil, según la Orden de 20 de mayo de 2000, por la que se regula dicha impartición». (BOC núm. 116, viernes 30 de agosto). Recuperado de http://www.gobiernodecanarias.org/boc/2002/116/011.html.

Gobierno de Canarias (2003). «Orden de 30 de junio de 2003, por la que se modifica la Orden de 20 de mayo de 2002, que regula la implantación de la enseñanza del inglés como primer idioma partir del segundo ciclo de la Educación Infantil». (Consejería de Educación, Cultura y Deportes, $B O C$ núm. 134, lunes 14 de julio). Recuperado de http://www. gobiernodecanarias.org/boc/2003/134/boc-2003-134-003.pdf.

Gobierno de Canarias (2015). PILE. Plan de Impulso de las Lenguas Extranjeras. Recuperado de https://www.gobiernodecanarias.org/cmsweb/export/sites/educacion/web/_galerias/ descargas/proyectos_legislativos/plan_impulso_lenguas_extranjeras_pile.pdf.

Haznedar, B. (2015). "Cognitive and linguistic aspects of learning a second language in the early years", en S. Mourao y M. Lourenço (eds.), Early years second language education. International perspectives on theory and practice (pp. 15-28). New York: Routledge.

Johnstone, R. (2002). Addressing «the age factor»: some implications for language policy. Guide for the development of language education policies in Europe. From linguistic diversity to plurilingual education. Recuperado de https://www.scilt.org.uk/Portals/24/Library/publications/ SCILT_2002_Johnstone_AddressingAgeFactor.pdf.

Krashen, S.D., Long, M.H. y Scarcella, R.C. (1982). «Age, rate and eventual attainment in second language acquisition", en S.D. Krashen, R.C. Scarcella y M.H. Long (eds.), Child-Adult differences in second language acquisition (pp. 161-172). Rowley, Massachusetts: Newbury House Publishers, Inc.

Krashen, S.D. (1986). Principles and practice in Second Language Acquisition. Oxford, England: Pergamon Press Ltd.

Kersten, K. (2015). «Bilingual pre-primary schools: language acquisition, intercultural encounters and environmental learning», en S. Mourao y M. Lourenço (eds.), Early years second language education. International perspectives on theory and practice (pp. 29-45). New York: Routledge.

Lourenço, M. y ANDrade, A.I. (2015). «Languages and diversity in pre-primary education: towards a broader and integrated approach», en S. Mourao y M. Lourenço (eds.), Early years second language education. International perspectives on theory and practice (pp. 120-136). New York: Routledge.

Lungu, I. (2015). «A new model of professionalisation of teachers in pre-school and primary school education». Social and Behavioural Sciences, 180: 632-638. DOI:10.1016/j.sbspro.2015.02.171.

Malkina, N. (2008). «Teaching foreign languages to young children in the Russian Federation", en R. Kantelinen, E.L. Sokka-Meaney y V. Pogosian (eds.), Seminar papers on early foreign language 
education (pp. 23-36). Recuperado de http://epublications.uef.fi/pub/urn_isbn_978-952219-188-5/urn_isbn_978-952-219-188-5.pdf.

Mourao, S. y Ferreirinha, S. (2016). Early language learning in pre-primary education in Portugal. Report, Associaçao Portuguesa de Professores de Inglés. Recuperado de https://www.appi.pt/ activeapp/wp-content/uploads/2016/07/Pre-primary-survey-report-July-FINAL-rev.pdf.

Mourao, S. y Lourenço, M. (eds.) (2016). Early years second language education. International perspectives on theory and practice. New York: Routledge.

Nikolov, M. y Djigunovic, J.M. (2006). «Recent research on age, second language acquisition, and early foreign language learning». Annual Review of Applied Linguistics, 26: 234-260. DOI: $10.1017 /$ S0267190506000122.

Scarcella, R.C. e Higa, C. (1982). "Input and age differences in second language acquisition», en S.D. Krashen, R.C. Scarcella y M.H. Long (eds.), Child-Adult differences in second language acquisition (pp. 175-201). Rowley, Massachusetts: Newbury House Publishers, Inc.

Seliger, H.W., Krashen, S.D. y Ladefoged, P. (1982). «Maturational constraints in the acquisition of second languages», en S.D. Krashen, R.C. Scarcella y M.H. Long (eds.), Child-Adult differences in second language acquisition (pp. 13-19). Rowley, Massachusetts: Newbury House Publishers, Inc.

Snow, C.E. y Hoefnagel-Höhle, M. (1982a). «Age differences in the pronunciation of foreign sounds", en S.D. Krashen, R.C. Scarcella y M.H. Long (eds.), Child-Adult differences in second language acquisition (pp. 84-92). Rowley, Massachusetts: Newbury House Publishers, Inc.

Snow, C.E. y Hoefnagel-Höhle, M. (1982b). «The critical period for language acquisition: evidence from second language learning», en S.D. Krashen, R.C. Scarcella y M.H. Long (eds.), Child-Adult differences in second language acquisition (pp. 93-111). Rowley, Massachusetts: Newbury House Publishers, Inc.

Wood, D., Bruner, J.S. y Ross, G. (1976). «The role of tutoring in problem-solving». Journal of Child Psychology and Psychiatry, 17: 89-100. DOI: 10.111/j.1469-7610.1976.tb00381.x. 


\begin{abstract}
ANEXO 1
TABLA DE FRECUENCIAS SEgúN COMPETENCIA LINGÜÍstica

8. Mi competencia en la lengua extranjera me permite entender, hablar, leer y escribir la lengua extranjera sin problemas.
\end{abstract}

11. Mi competencia en la lengua extranjera es suficiente para enseñarla a edades tempranas.

TABLA DE FRECUENCIAS SEGÚN COMPETENCIA PEDAGógica

1. Los niños aprenden una lengua extranjera más fácilmente que los adultos.

3. El aprendizaje de lenguas extranjeras a edades tempranas puede tener un impacto negativo en la adquisición de la lengua materna.

4. Cuanto antes se introduzca la lengua extranjera en los programas escolares, mayores serán las posibilidades de éxito en su aprendizaje.

5. La enseñanza de lenguas extranjeras demanda no solo conocimiento de la lengua, sino de las metodologías y el currículo específicos de la etapa.

6. Un enfoque inadecuado en la enseñanza de la lengua extranjera podría causar efectos negativos en el aprendizaje y la motivación de los escolares.

7. No es necesario contar con una buena competencia en la lengua extranjera para enseñarla a los niños porque sus necesidades comunicativas son muy básicas.

TABLA DE FRECUENCIAS SEGÚN COMPETENCIA PROFESIONAL

9. Me gustaría enseñar la lengua extranjera en esta etapa.

10. Estaría dispuesto a realizar cursos de perfeccionamiento en la lengua y en la metodología para su enseñanza, en el futuro.

12. Es improbable que los docentes de esta etapa puedan que enseñar la lengua a los niños.

13. Los docentes de Infantil no deberían enseñar la lengua extranjera a los niños de esta etapa porque no es su especialidad.

TABLA DE FRECUENCIAS SEGÚN COMPETENCIA INTERCULTURAL

2. El aprendizaje precoz de lenguas extranjeras ayuda a aprender sobre otras culturas y a aceptarlas. 